\begin{tabular}{|c|l|}
\hline Title & Simulation studies of photon-assisted quantum transport \\
\hline Author(s) & Y akubo, Kousuke \\
\hline Citation & $\begin{array}{l}\text { PHY SICAL REVIEW B, 54(11), 7987-7995 } \\
\text { https://doi.org/10.1103/PhysRevB.54.7987 }\end{array}$ \\
\hline Issue Date & 1996-09-15 \\
\hline Doc URL & http://hdl.handle.net/2115/5654 \\
\hline Rights & Copyright $\odot$ 1996 A merican Physical Society \\
\hline Type & article \\
\hline File Information & PRB54 11.pdf \\
\hline
\end{tabular}

Instructions for use 


\title{
Simulation studies of photon-assisted quantum transport
}

\author{
Kousuke Yakubo \\ Department of Applied Physics, Hokkaido University, Sapporo 060, Japan \\ Shechao Feng* \\ Department of Physics, University of California, Los Angeles, California 90024 \\ Qing $\mathrm{Hu}$ \\ Department of Electrical Engineering and Computer Science and Research Laboratory of Electronics, \\ Massachusetts Institute of Technology, Cambridge, Massachusetts 02139 \\ (Received 17 April 1995; revised manuscript received 21 December 1995)
}

\begin{abstract}
Direct numerical simulations were performed to investigate conditions for the strong effects of photonassisted quantum transport. Transmission probabilities of incident electrons as Gaussian wave packets were calculated for single- and double-barrier structures irradiated by electromagnetic fields that were focused within a finite region $\xi_{\mathrm{ac}}$. When the period of the ac electromagnetic field is short compared with the time $\tau_{\xi_{\text {ac }}}$, which is the traversal time of the electrons in the region of the applied ac field, the transmission probability can be affected by photon emission and photon absorption. The magnitude of these effects strongly depends on the width $\xi_{\mathrm{ac}}$ and the bound-state character of the electrons. These dependences can be understood from the selection rule, which results from the momentum conservation of the electron and photon system. Consequently, we have shown that photon-assisted transport over a single barrier is difficult to achieve in two-dimensional electron-gas devices due to the relatively long screening length. We have demonstrated, however, that the photon-assisted process is significantly enhanced in double-barrier devices due to quasibound-to-extended-state transitions. [S0163-1829(96)02435-6]
\end{abstract}

\section{INTRODUCTION}

Photon-assisted transport at far-infrared frequencies $(\hbar \omega \approx 1-10 \mathrm{meV})$ in quantum-point-contact devices has been proposed ${ }^{1}$ and theoretically investigated. ${ }^{2}$ In essence, the process of photon-assisted transport is analogous to the photoemission phenomenon in metals, which inspired Einstein to propose the famous $E=\hbar \omega$ relationship. Our quantitative analysis of the photon-assisted transport process is based on Tien-Gordon's theory of photon-assisted tunneling in superconducting tunnel junctions. ${ }^{3}$ In this theory, photon absorption (or emission) is characterized as creating a set of electron eigenstates with eigenenergies of $E+n \hbar \omega$, where $E$ is the eigenenergy of the original electron state without radiation. Positive $n$ 's correspond to photon absorption, while negative $n$ 's correspond to photon emission. The probability of absorbing or emitting $n$ photons is proportional to $J_{n}^{2}(\alpha)$, where $J_{n}$ is the $n$th Bessel function and $\alpha$ is a dimensionless number that is proportional to the radiation field strength. Consequently, similar to photon-assisted tunneling steps for superconducting tunnel junctions, photon-assisted steps for quantum-point-contact devices should appear on the drain-source conductance vs the gate voltage $G_{\mathrm{DS}}-V_{\mathrm{GS}}$ curves, provided that the photon energy is much greater than the thermal broadening.

In spite of the intuitively plausible picture and the straightforward analogy with photoemission and photonassisted tunneling, experimental investigations have failed to provide evidence of photon-assisted transport in quantumpoint-contact devices. ${ }^{4,5}$ Extensive measurements conducted over a broad frequency range from $90 \mathrm{GHz}$ to $2.5 \mathrm{THz}$
( $\hbar \omega \approx 0.3-10 \mathrm{meV}$ ), with the electric-field polarization both parallel and perpendicular to the drain-source conductance path, have yielded only bolometric signals. These signals result from heating of the 2DEG (two-dimensional electron gas) in the source and drain. ${ }^{4,5}$ The process of photonassisted quantum transport is analogous to many wellestablished phenomena such as photoemission in metals, ${ }^{6}$ photoionization of atoms, ${ }^{7-9}$ photon-excited bound-toextended-state transitions in quantum-well structures, ${ }^{10}$ and photon-assisted tunneling in quantum-well diodes, ${ }^{11}$ superconducting tunnel junctions, ${ }^{3,12-15}$ and quantum dots. ${ }^{16,17}$ Thus it is natural to ask why such a simple process has not been observed in quantum-point-contact devices. It is the scope of this paper to solve the one-dimensional Schrödinger equation numerically, including the radiation term, and to investigate the conditions necessary for photon-excited processes as characterized by additional components at energy $E+n \hbar \omega$ in the wave function. These conditions are a direct consequence of the selection rule, which is a mathematical statement of the momentum conservation for electron-photon systems. Mathematically, in order to achieve an appreciable photon-excited transition probability, the dipole-moment integration must be truncated to a region that is not much greater than the coherence length $1 / \Delta k$, where $\Delta k$ is equal to the momentum difference between the electrons before and after photon absorption or emission. This truncation can be realized experimentally by either a localized electron state or a localized photon-field profile. Physically, this spatial localization of the electron or photon field provides the momentum spread that relaxes the requirement for momentum conservation in photon-excited processes. Without such a spatial 
localization, the probability of photon excitation will be exponentially small. Thus heating, which is unavoidable because of the gapless nature of excitations in 2DEG, will dominate the radiation response of the devices.

As a consequence of this understanding, we have found that the photon-excited transition between two extended electronic states is prohibited if the radiation field is spatially extended. Therefore, photon-assisted transport in singlebarrier devices, such as quantum point contacts, ${ }^{1,2,4,5,18}$ will be difficult to observe due to the relatively long screening length in 2DEG structures. For superconducting tunnel junctions, photon-assisted tunneling does occur between two extended electron states. In this case, the strong screening process localizes the photon field near the tunnel barriers within the London penetration depth, thus enabling the transition to occur., ${ }^{3}-15$ For $2 \mathrm{DEG}$ devices, we have demonstrated that double-barrier (or multiple-barrier) structures are more suitable for photon-assisted transport, which results from the quasibound-to-extended-state transitions. This theoretical understanding is supported by strong evidence of photonassisted transport observed in a dual-gate device irradiated at millimeter-wave frequencies. ${ }^{19}$

This paper is organized as follows. In Sec. II we describe a numerical path-integral technique used to solve the timedependent Schrödinger equation. Effects of wave packets with finite widths are also discussed. In Sec. III, conditions for strong quantum coupling between electrons and electromagnetic fields are shown by calculating the escape probability of an electron from a bound state by photon excitations. We also discuss the coupling between free Gaussian wave packets and spatially confined ac fields. By calculating the transmission probabilities through single-barrier structures irradiated by electromagnetic fields, it is shown that photon-assisted transport over a single barrier is difficult to achieve in 2DEG devices, unless a tightly confined ac field can be produced. In Sec. IV, we calculate transmission probabilities for double-barrier structures and present numerical evidence of photon-assisted quantum transport that is shown as an enhancement of transmission probability. We summarize our conclusions in Sec. V.

\section{NUMERICAL TECHNIQUE}

We have investigated transport properties of electrons in one-dimensional systems with both time-independent and time-dependent potentials. These potentials correspond to 2DEG devices that have some structure in the $x$ direction, but remain uniform in the $y$ direction. It can be shown that quantum-point-contact systems that have structures in both $x$ and $y$ directions can be reduced to one-dimensional systems. ${ }^{2}$ Therefore, our focus on one-dimensional systems does not sacrifice generality, but it does significantly reduce the mathematical complexity. An electron in such a system is governed by the time-dependent Schrödinger equation

$$
i \frac{\partial \psi}{\partial t}=\left[-\frac{1}{2 m^{*}} \frac{\partial^{2}}{\partial x^{2}}+V_{\mathrm{dc}}(x)+V_{\mathrm{ac}}(x, t)\right] \psi,
$$

where $V_{\mathrm{dc}}(x)$ is a time-independent static potential, $V_{\text {ac }}(x, t)=\int_{0}^{x} e \mathcal{E}\left(x^{\prime}, t\right) d x^{\prime}$ is a time-dependent potential that represents the effect of a radiation field, $\mathcal{E}(x, t)$ is a time- dependent electric field, and $e$ and $m^{*}$ are the charge and the effective mass of an electron, respectively. Here we use the units of $\hbar=1$. It should be noted that Eq. (2.1) also describes electrons in three-dimensional layered structures grown by molecular-beam epitaxy. In this case, the polarization of the electromagnetic field and the tunneling current are in the growth" direction. Most of resonant-tunneling experiments ${ }^{10,20-29}$ belong to this configuration. The only difference between 2DEG configurations and layered structures is the typical values of parameters such as electron densities and dimensions of dc potential profiles. Here we employ parameter values that correspond to 2DEG configurations. In order to solve Eq. (2.1) and calculate transmission probabilities, we choose a Gaussian wave packet as the incident electron wave function. This initial condition is relevant to actual experiments at finite temperatures. The temperatures at which quantum transport experiments are performed are typically between 0.1 and $10 \mathrm{~K}$. Thus the energy of incident electrons has a finite spread. The effect of this energy uncertainty can be represented by incident Gaussian wave packets given by

$$
\psi(x, 0)=\left(2 \pi \delta^{2}\right)^{-1 / 4} \exp \left[-\frac{(x-X)^{2}}{4 \delta^{2}}+i k_{0} x\right],
$$

where $\delta$ is the width of the wave packet related to the temperature $T$ and the Fermi energy $E_{\mathrm{F}}$ through $k_{\mathrm{B}} T \delta=\sqrt{2 E_{\mathrm{F}} / m^{*}}$, and $k_{0}$ is the wave number of the incident electron given by

$$
k_{0}=\left[2 m * E_{\mathrm{F}}-\frac{1}{2 \delta^{2}}\right]^{1 / 2} .
$$

We have employed a numerical path integral method ${ }^{30}$ to solve Eq. (2.1) numerically. Using this technique, the condition of the unitary in a time development is automatically satisfied, regardless of the values of the time step $\tau$ and the grid spacing of spatial division $\Delta_{x}$. Furthermore, this algorithm demands a relatively short computing time to solve the equation for large systems such as those treated here, as compared with other conventional methods. ${ }^{31-38}$

It is well known that the wave function $\psi(x, t+\tau)$ is related to $\psi(x, t)$ through

$$
\psi(x, t+\tau)=\int d y K(x, y ; t, \tau) \psi(y, t),
$$

where the propagator $K(x, y ; t, \tau)$ is given in the path integral formalism by ${ }^{39}$

$$
K(x, y ; t, \tau)=\int_{y}^{x} \mathcal{D} q \exp \left[i \int_{t}^{t+\tau} d t^{\prime} L\left(\dot{q}\left(t^{\prime}\right), q\left(t^{\prime}\right) ; t^{\prime}\right)\right] .
$$

Here the symbol $\int_{y}^{x} \mathcal{D} q$ represents the path integral over all paths, starting at the point $y$ and ending with $x$, and $\mathcal{D} q$ is a measure for integration defined by

$$
\mathcal{D} q \equiv \lim _{N \rightarrow \infty}\left(\frac{1}{2 \pi \tau i}\right)^{N / 2^{N-1}} \prod_{j=1} d q_{j} .
$$




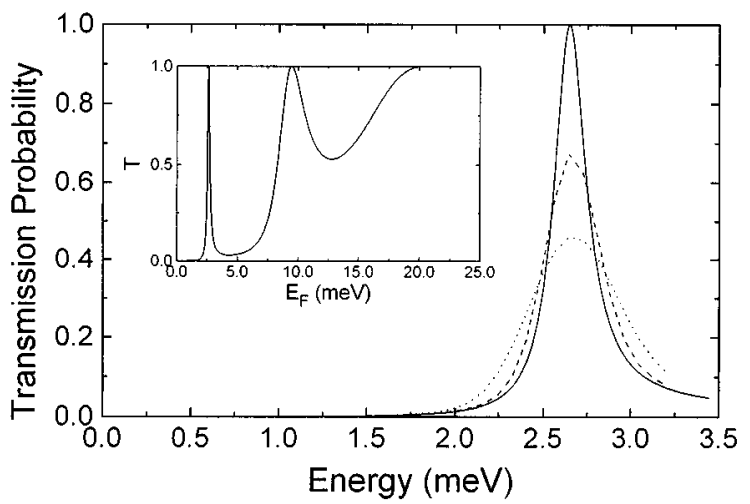

FIG. 1. Transmission probability for a symmetric double-barrier structure (with $V_{\mathrm{dc}}^{0}=10 \mathrm{meV}, 2 x_{0}=433 \AA$, and $\xi_{\mathrm{dc}}=72 \AA$ ) as a function of the energy of the incident electrons. Solid lines in this figure and its inset indicate the transmission probability calculated from the transfer-matrix method. Dotted and dashed lines indicate the results calculated from the numerical path-integral method using incident wave packets with a width of 1805.5 and $3611.0 \AA$, respectively.

The integrand $L(\dot{q}, q ; t)$ in Eq. (2.5) is the Lagrangian of the system, and has the form

$$
L(\dot{q}, q ; t)=\frac{1}{2} m^{*} \dot{q}^{2}-V_{\mathrm{dc}}(q)-V_{\mathrm{ac}}(q, t) .
$$

To perform the path integral numerically, one needs to consider the case that the value of $\tau$ is so small that only the straight path $s$ from $y$ to $x$ makes a dominant contribution to the path integration. In this case, the integration of $V\left(q_{s}\left(t^{\prime}\right), t^{\prime}\right) \equiv V_{\mathrm{dc}}\left(q_{s}\left(t^{\prime}\right)\right)+V_{\mathrm{ac}}\left(q_{s}\left(t^{\prime}\right), t^{\prime}\right)$ over $t^{\prime}$ in Eq. (2.5) becomes approximately $\tau V(x, t+\tau)$. This quantity no longer depends on the path; thus the potential energy gives only the factorial contribution to the propagator $K(x, y ; t, \tau)$. The path integral of the kinetic energy part can be evaluated analytically. ${ }^{39}$ Finally, Eq. (2.4) is approximated as

$$
\begin{aligned}
\psi(x, t+\tau)= & \left(\frac{m^{*}}{2 \pi \tau i}\right)^{1 / 2} \exp [-i \tau V(x, t+\tau)] \\
& \times \int_{-\infty}^{\infty} \exp \left[\frac{i m^{*}(x-y)^{2}}{2 \tau}\right] \psi(y, t) d y .
\end{aligned}
$$

Using this relation iteratively, one can calculate the wave function at any time $t$ from the initial wave function $\psi(x, 0)$. By calculating the normalization factor of $\psi(x, t+\tau)$, one can show that this procedure satisfies the unitary condition for any time steps $\tau$ and spatial divisions $\Delta_{x}$. In actual numerical calculations, the convolution in Eq. (2.8) was done in the Fourier space in order to save CPU time.

In order to illustrate the effect of the finite width of wave packets associated with actual experiments at finite temperatures, we compare the transmission probability calculated by the numerical path-integral method described above with those obtained by the conventional transfer-matrix technique, which corresponds to the transmission for plane waves. Figure 1 shows the transmission probability for the following symmetric double-barrier structure as a function of energy of the incident wave,

$$
V_{\mathrm{dc}}(x)=V_{\mathrm{dc}}^{0}\left\{\exp \left[-\frac{\left(x-x_{0}\right)^{2}}{\xi_{\mathrm{dc}}^{2}}\right]+\exp \left[-\frac{\left(x+x_{0}\right)^{2}}{\xi_{\mathrm{dc}}^{2}}\right]\right\},
$$

where the barrier height $V_{\mathrm{dc}}^{0}$ is $10 \mathrm{meV}$, the distance between two peaks $2 x_{0}$ is $433 \AA$, and the width of each Gaussian barrier $\xi_{\text {dc }}$ is $72 \AA$. In this case, no ac potential is applied to the system. The solid lines in Fig. 1 and its inset show the result obtained by the transfer-matrix method, in which the energy of the incident electron has a definite value. Here and hereafter we have chosen the material parameter corresponding to GaAs, $m^{*}=0.067 m_{0}$, where $m_{0}$ is the bare electron mass. The sharper peak in the inset represents the resonant tunneling due to the quasibound state. This peak is magnified in Fig. 1. The dashed and the dotted lines are the results of the numerical path-integral method. The widths of the incident Gaussian wave packets for the dashed and dotted lines are 3611 and $1805 \AA$, respectively. The energy fluctuations of these wave packets are 0.21 and $0.41 \mathrm{meV}$, which correspond to temperatures of 2.38 and $4.75 \mathrm{~K}$, respectively. From this figure, we can appreciate the importance of using the finite-width wave packet to approximate actual experimental situations at finite temperatures. For these calculations, we have chosen a system size of $23.66 \mu \mathrm{m}$ to accommodate the transmitting and reflecting waves going far from the origin during long transit times due to the quasibound state. In order to obtain precise results, the spatial division $\Delta_{x}$ should be made much smaller than the scale of both the potential structure and the incident wavelength $k_{0}^{-1}$. Here the value of $\Delta_{x}=3.6 \AA$ is used. Thus the number of grids in the system is $32768\left(=2^{15}\right)$. The time step $\tau$ must be much smaller than $1 / E_{\mathrm{F}}$. We chose $\tau=15.7 \mathrm{fs}$. Since the transmission process takes about 23 ps to complete for this particular doublebarrier structure, we need about 1400 time steps in our simulation.

\section{CONDITIONS FOR QUANTUM EXCITATIONS}

In this section, we discuss conditions required to obtain strong quantum interactions between electrons and radiation fields, and the design of realizable systems to measure these effects efficiently. For this purpose, this section focuses on the interaction between electrons and electromagnetic fields beyond simple tunneling phenomena.

Due to the selection rule, a free electron described by a plane wave cannot interact with a uniform electromagnetic field. The matrix element for this interaction has a factor $\int x \exp \left[i\left(k-k^{\prime}\right) x\right] d x$, where $k$ and $k^{\prime}$ are the wave number of the initial and final electron states. This factor is proportional to the derivative $\delta^{\prime}\left(k-k^{\prime}\right)$, and it is always zero for $k \neq k^{\prime}$. Thus the transition between $k$ and $k^{\prime}$ that is excited by a spatially uniform field cannot occur. This selection rule is simply a mathematical statement that says free electrons and photons have different energy-momentum dispersion relations, and therefore it is impossible to satisfy the energy and momentum conservations simultaneously, except at a zero frequency. This is known as the generalized Kohn's theorem. We can show that the selection rule also applies to 
free-electron states other than the plane waves. For example, a free Gaussian wave packet in a uniform electromagnetic field will not develop photon sidebands. The solution of Schrödinger equation (2.1) with $V_{\mathrm{dc}}(x)=0$ and $V_{\mathrm{ac}}(x, t)=$ $-e \mathcal{E} x \cos (\omega t)$ can be written in terms of the nonrelativistic Gordon-Volkov function ${ }^{40-42}$

$$
\psi_{k}(x, t)=\frac{1}{\sqrt{2 \pi}} \exp \left[i \widetilde{k x}-\frac{i}{2} \int_{0}^{t} d t^{\prime} \widetilde{k}^{2}\left(t^{\prime}\right)\right]
$$

where the reduced momentum $\tilde{k}$ is defined by $\widetilde{k}=k+(e \mathcal{E} / \omega) \sin (\omega t)$, and $k$ is the electron momentum. Using this complete basis set and the initial condition given by Eq. (2.2) with $X=0$ and $k_{0}=0$, we can obtain an expression for the electron probability density:

$$
|\psi(x, t)|^{2}=\left(\frac{2}{\pi \Delta^{2}(t)}\right)^{1 / 2} \exp \left\{-\frac{\left[x-x_{c}(t)\right]^{2}}{2 \Delta^{2}(t)}\right\},
$$

where

$$
\Delta(t)=\frac{\sqrt{2 \delta^{4}+\left(t^{2} / m^{* 2}\right)}}{\delta},
$$

and the center-of-mass motion $x_{c}(t)$ has the classical trajectory of the electron in the same electromagnetic field. Quantum-mechanical spreading of the wave packet is not influenced by the uniform ac electromagnetic field, which is evidenced by the fact that $\Delta(t)$ is independent of the ac field $\mathcal{E}$. This analysis indicates that there is no quantum interaction between a free Gaussian wave packet and a uniform ac field.

From the form of a coupling matrix element, either nonuniform ac fields and/or electrons in bound states can lead to finite excitation probabilities. We will examine these conditions separately. The interaction between an uniform electromagnetic field and an electron in a bound state is treated first. A typical example is the ionization of atoms in laser fields. ${ }^{7-9}$ When the wavelength of the laser field is much longer than the spatial spreading of the electron wave function bound to an atom, the ionization is a result of the interaction between the localized electron and a uniform field.

In order to understand quantitatively the photon excitations, we have numerically investigated the ionization process in a uniform ac electric field. We have considered the case in which an electron is initially in a one-dimensional potential well given by

$$
U=-U_{0} \operatorname{sech}^{2}(\alpha x),
$$

where $U_{0}=7.303 \mathrm{meV}$ and $\alpha^{-1}=250 \AA$. These parameters are not relevant to atomic scales, but close to mesoscopic ones. Time-independent Schrödinger equation with this potential can be solved analytically. ${ }^{43}$ For the given set of parameters $U_{0}$ and $\alpha\left(U_{0}=35 \alpha^{2} / 8 m^{*}\right)$, there are three bound states. The energies of these states are

$$
E_{n}=-\frac{(5-2 n)^{2} \alpha^{2}}{8 m^{*}}, \quad n=0,1, \text { and } 2,
$$

with their values of $E_{0}=-5.2164 \mathrm{meV}, E_{1}=-1.8779 \mathrm{meV}$, and $E_{2}=-0.2086 \mathrm{meV}$. The corresponding eigenstates are given by

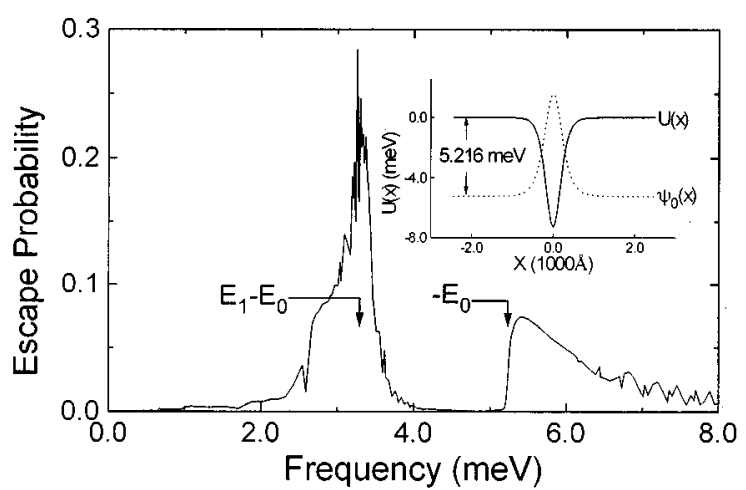

FIG. 2. Escape probability of an electron from the potential well $U(x)$ given by Eq. (3.3) under the irradiation of a uniform electromagnetic field $\mathcal{E} \cos (\omega t)$ as a function of $\omega$. The strength of the field is $e \mathcal{E} / \alpha U_{0}=0.0488$. The irradiation time $T$ is $75.63 \mathrm{ps}$. The inset shows the configuration of the potential well $U(x)$ and the ground state (which is the initial state) wave function $\psi_{0}(x)$.

$$
\begin{gathered}
\psi_{0}=A_{0}\left[1-\tanh ^{2}(\alpha x)\right]^{5 / 4}, \\
\psi_{1}=A_{1}\left[1-\tanh ^{2}(\alpha x)\right]^{3 / 4} \tanh (\alpha x),
\end{gathered}
$$

and

$$
\psi_{2}=A_{2}\left[1-\tanh ^{2}(\alpha x)\right]^{1 / 4}\left[4 \tanh ^{2}(\alpha x)-1\right],
$$

respectively, where $A_{0}, A_{1}$, and $A_{2}$ are normalization constants. We choose the ground state $\psi_{0}$ as the initial state. The configuration of the potential and the ground-state wave function are illustrated in the inset of Fig. 2. We calculate numerically the time development of this bound state under a uniform electromagnetic field described by an ac potential $V_{\text {ac }}(x, t)=-e \mathcal{E} x \cos (\omega t)$. The strength of the field $e \mathcal{E}$ was chosen as $e \mathcal{E} / \alpha U_{0}=0.0488$. The system size, the space division, and the time step in this calculation are $5.12 \mu \mathrm{m}, 50 \AA$, and $15.3 \mathrm{fs}$, respectively. Figure 2 shows the escape probability from the potential well plotted as a function of frequency of the ac field. The escape probability $P_{E}$ was defined by

$$
P_{E}=\int_{x_{\mathrm{L}}}^{x_{1}}|\psi(x, T)|^{2} d x+\int_{x_{2}}^{x_{\mathrm{R}}}|\psi(x, T)|^{2} d x,
$$

where $x_{\mathrm{L}}$ and $x_{\mathrm{R}}$ are the left- and right-side edges, respectively. The values of $x_{1}$ and $x_{2}$ were set as $x_{1}=-2500 \AA$ and $x_{2}=2500 \AA$. The irradiation time $T$ was chosen as $T=75.63 \mathrm{ps}$. Within this time interval, the finite-size effect can be neglected. From Fig. 2, one can find a sharp onset at the ionization energy $-E_{0}=5.22 \mathrm{meV}$, which shows that the electron in the bound state couples strongly with the uniform ac field. By increasing the photon energy from $-E_{0}$, the escape probability decreases rapidly. This is because the overlap integral between the initial (ground) state and the excited extended state becomes smaller at higher frequencies. There is also a sharp peak at $3.3 \mathrm{meV}$. This energy is the same as the energy difference between $E_{0}$ and $E_{1}$, which means that the peak is caused by a two-photon process. Indeed, the Fourier transform of the outgoing wave from the potential well has a sharp peak at $1.4 \mathrm{meV}$, which is equal to 


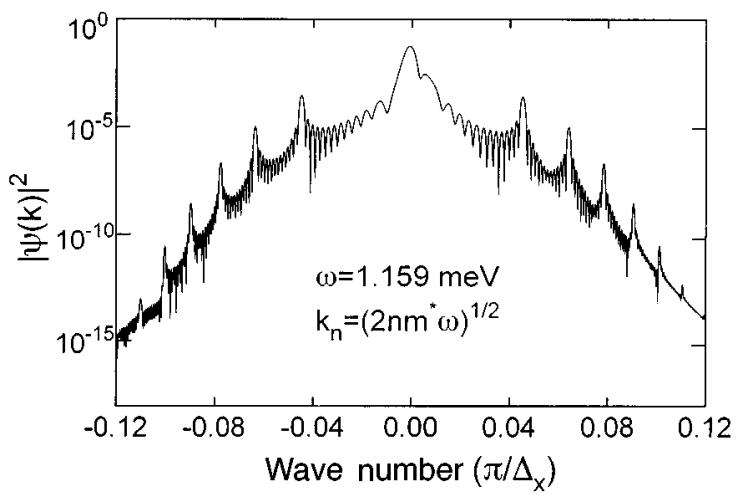

FIG. 3. Square of the Fourier transform of the wave function at $t=43.56 \mathrm{ps}$ of a free electron irradiated by a spatially confined electromagnetic field. The initial wave function is a Gaussian wave packet described by Eq. (2.2) with $\delta=3000 \AA, X=0$, and $k_{0}=0$. The applied ac potential is given by Eq. (3.6) with $V_{\mathrm{ac}}^{0}=1.159 \mathrm{meV}$, $\xi_{\mathrm{ac}}=600 \AA$, and $\omega=1.159 \mathrm{meV}$.

$E_{0}+2\left(E_{1}-E_{0}\right)$. The strong two-photon effect is a result of a large overlap integral between the ground state and the first excited state, and between the first excited state and the extended state over the barriers.

Next, we discuss interactions between a free electron and a nonuniform electromagnetic field. It was previously mentioned that a free electron cannot interact with a uniform ac field due to the selection rule. The matrix element of the coupling, however, becomes nonzero if the electromagnetic field is not uniform, and the probability of photon absorption becomes finite. In order to illustrate this point quantitatively, we studied interactions between a free Gaussian wave packet at rest and a spatially confined electromagnetic field. Fourier transforms of the wave packet under the ac field are calculated. The initial wave packet is given by Eq. (2.2) with $X=0, k_{0}=0$, and $\delta=3000 \AA$. The confined electromagnetic field is described by a time-dependent (ac) potential

$$
V_{\mathrm{ac}}(x, t)=\frac{V_{\mathrm{ac}}^{0}}{2} \tanh \left(\frac{x}{\xi_{\mathrm{ac}}}\right) \cos (\omega t) .
$$

The parameters in Eq. (3.6) were chosen as $V_{\mathrm{ac}}^{0}=1.159 \mathrm{meV}$, $\xi_{\mathrm{ac}}=600 \AA$, and $\omega=1.159 \mathrm{meV}$. The system size, the space division, and the time step are $24.58 \mu \mathrm{m}, 30 \AA$, and $2.72 \mathrm{fs}$, respectively. The square of the Fourier transform of the wave function at $t=43.56 \mathrm{ps}$ (irradiation starts at $t=0),|\psi(k)|^{2}$, is shown in Fig. 3. The main peak at $k=0$ represents the original Gaussian wave packet. One can easily find at least five pairs of subpeaks located symmetrically around the main peak. These subpeaks are at the wave numbers $k_{ \pm 1}=$ $\pm 0.043, k_{ \pm 2}= \pm 0.061, k_{ \pm 3}= \pm 0.075, k_{ \pm 4}= \pm 0.086$, and $k_{ \pm 5}= \pm 0.097$ in units of $\pi / \Delta_{x}$, where $\Delta_{x}$ is the grid spacing of the spatial division. These values correctly correspond to $k_{ \pm n}= \pm \sqrt{2 n m^{*} \omega}$, which is the wave number of the excited electrons produced by the $n$-photon process. Thus these subpeaks are referred to as photon subbands. Clearly, a spatially confined electromagnetic field can interact effectively with free electrons.

We have investigated the effect of the width of the confined ac field on photon-absorption processes. Figure 4 shows the values of $|\psi(k)|^{2}$ at one-, two-, and three-photon

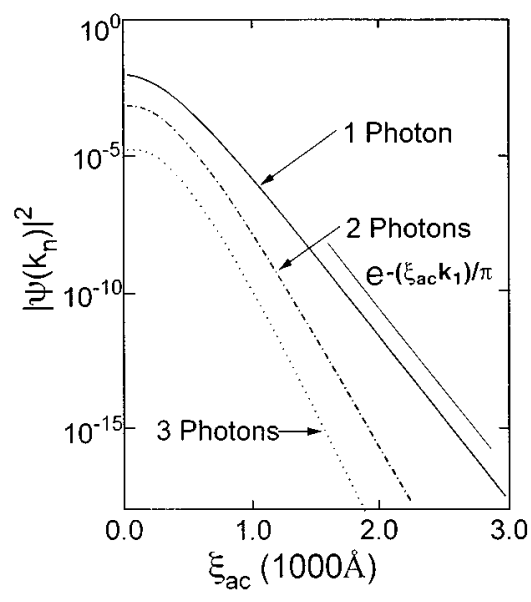

FIG. 4. Peak values of $|\psi(k)|^{2}$ as functions of the width of the ac field $\xi_{\text {ac }}$. Parameters for the calculations are the same as those for Fig. 3, except for the irradiation time of 21.78 ps and the system size. Solid, broken, and dotted lines indicate the values of the photon subbands $|\psi(k)|^{2}$ at $k=0.043,0.061$, and 0.075 , respectively. The straight line in the semilog plot indicates the exponential relation of $\left|\psi\left(k_{1}\right)\right|^{2} \propto \exp \left(-\xi_{\mathrm{ac}} k_{1} / \pi\right)$.

absorption peaks as functions of the spatial confinement $\xi_{\text {ac }}$ of the applied ac field. Parameters in the calculation are the same as those for Fig. 3, except for the system size of 12.29 $\mu \mathrm{m}$ and the radiating time of 21.78 ps. This figure clearly shows that the interaction between a free electron and an electromagnetic field decreases exponentially as the width $\xi_{\mathrm{ac}}$ of the confined ac field increases, while the value of $V_{\mathrm{ac}}^{0}$ is kept constant. The straight line in the semilog plot shows the asymptote of $\left|\psi\left(k_{1}\right)\right|^{2}$ at large $\xi_{\text {ac }}$ values, which has a functional form of $e^{-\left(\xi_{\mathrm{ac}} k_{1}\right) / \pi} \cdot\left|\psi\left(k_{2}\right)\right|^{2}$ and $\left|\psi\left(k_{3}\right)\right|^{2}$ decrease even faster with $\xi_{\mathrm{ac}}$ because of the larger values of $k_{2}$ and $k_{3}$. In the present numerical situation, the wavelength of the excited electron through a one-photon process is $1380 \AA$. It appears from Fig. 4 that the interaction decreases rapidly when $\xi_{\mathrm{ac}}>\lambda / 2 \pi \approx 200 \AA$. This result can be understood from an analytical argument. The matrix element of this interaction has a factor of $\int \exp \left(-x^{2} / 4 \delta^{2}\right) \exp (i k x) \tanh \left(x / \xi_{\text {ac }}\right) d x$, where $k$ is the wave number of the excited state. The real part of this function vanishes because of the integrand's parity. We need to consider only the imaginary part of this integral. For $\delta \gg 1 / k$, which is the case in this situation, $\sin (k x)$ oscillates many times within the Gaussian width. If the value of $\xi_{\text {ac }}$ is much larger than $1 / k$, this oscillation cancels out the integral. In the case of $\xi_{\mathrm{ac}} \lesssim 1 / k$, however, the oscillation cannot be canceled out within the range $|x| \lesssim \xi_{\mathrm{ac}}$, and the matrix element becomes finite. The wave number of the excited electron $k$ is related to the energy $\omega$ by $k=\sqrt{2 m^{*} \omega}$. Thus it is necessary for a large coupling that the value of $\xi_{\text {ac }}$ be less than $1 / \sqrt{2 m^{*} \omega}$. This means the coupling becomes weaker at higher frequencies for a fixed confinement $\xi_{\mathrm{ac}}$. This is essentially the same phenomenon as the decrease of the escape probability at higher frequencies (beyond $-E_{0}$ ) in Fig. 2. Similarly, we will also have a large matrix element if $\delta \lesssim 1 / k$, which qualitatively corresponds to the bound-toextended-state transition shown in Fig. 2. 


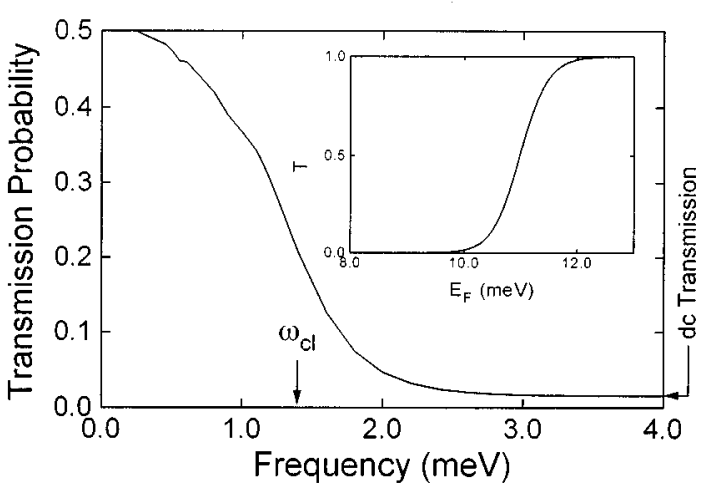

FIG. 5. Transmission probability for an irradiated single Gaussian potential barrier (with $V_{\mathrm{dc}}^{0}=11 \mathrm{meV}$ and $\xi_{\mathrm{dc}}=1000 \AA$ ) as a function of the frequency of the ac field. The incident wave packet has an energy of $10 \mathrm{meV}$ and a width of $12000 \AA$. The amplitude $V_{\mathrm{ac}}^{0}$ and the width $\xi_{\mathrm{ac}}$ of the ac potential are $10 \mathrm{meV}$ and $3000 \AA$, respectively. The crossover frequency $\omega_{\mathrm{cl}}$ from the classical to the quantum region is indicated by an arrow. The transmission probability for the nonirradiated single barrier with the same structure at $E_{\mathrm{F}}=10 \mathrm{meV}$ is also shown by an arrow. The inset shows the dc transmission probability for the same static potential as a function of the energy of the incident electron, which is calculated from the transfer-matrix method.

This argument can be also understood in terms of the uncertainty principle. According to this principle, a photon excitation process that results in an electron momentum change $\Delta k$ should occur within a spatial region on the order of $1 / \Delta k$. This limits $\xi_{\mathrm{ac}}<1 / \Delta k$. The quantity $\Delta k$ is evaluated as $\Delta k \sim \omega \sqrt{m^{*} / 2 E_{\mathrm{F}}}$ for $E_{\mathrm{F}} \gg \omega$, and $\Delta k \sim \sqrt{2 m^{*} \omega}$ for $E_{\mathrm{F}} \ll \omega$. In the case of Figs. 3 and $4, E_{\mathrm{F}}=0$ and $\Delta k=k_{1}$. Therefore, we have the condition $\xi_{\mathrm{ac}}<1 / \sqrt{2 m^{*} \omega}$ to obtain a strong coupling between a free electron at rest and an electromagnetic field. For $E_{\mathrm{F}} \gg \omega$, the condition becomes $\xi_{\mathrm{ac}}<\sqrt{2 E_{\mathrm{F}} / m^{*}} / \omega$.

We can conclude from these investigations that in order to obtain a strong coupling between an electron and an electromagnetic field, either the electrons must be in a bound or quasibound state or that the ac field must be confined within a spatial region of the order of $1 / \Delta k(\pi / \Delta k$ to be more precise). We believe this is the main reason that experiments on photon-assisted transport in $2 \mathrm{DEG}$ devices have so far yielded negative results in single-barrier structures such as quantum point contacts. In Fig. 5, our numerical simulation shows that only a weak photonic quantum effect appears in electron transport through a single-barrier structure. This figure shows the frequency dependence of the transmission probability affected by an electromagnetic field with a weakly confined spatial profile. The shape of the static potential barrier is given by

$$
V_{\mathrm{dc}}(x)=V_{\mathrm{dc}}^{0} \exp \left[-x^{2} / \xi_{\mathrm{dc}}^{2}\right]
$$

where the potential height $V_{\mathrm{dc}}^{0}$ is $11 \mathrm{meV}$ and the width $\xi_{\mathrm{dc}}$ is $1000 \AA$. The incident energy dependence of the transmission probability for the dc potential alone is shown in the inset of Fig. 5. The result of the frequency dependence shown in Fig. 5 is calculated for a fixed energy of the incident wave packet at $E=10 \mathrm{meV}$, which is the typical value of the Fermi en-

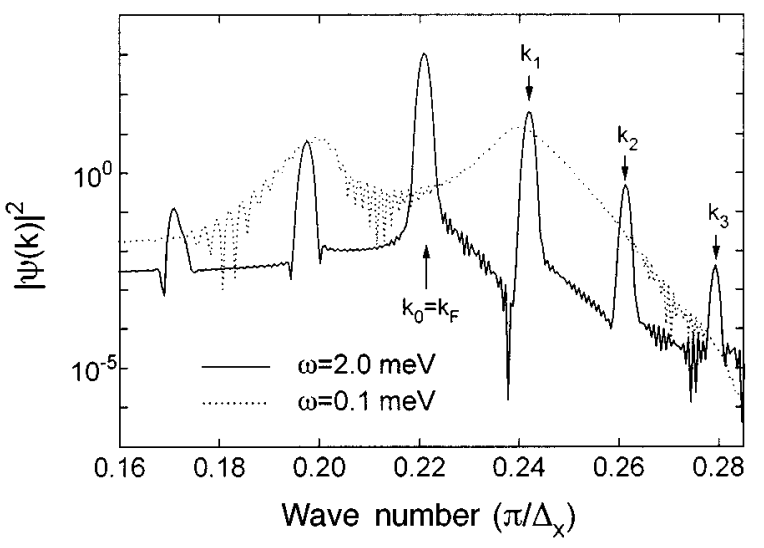

FIG. 6. Squares of Fourier transforms of the wave functions at $t=39.9$ ps for the same conditions with those for the case of Fig. 5 . The solid and dashed lines show the results for $\omega=2.0$ and 0.1 $\mathrm{meV}$, respectively. There are sharp peaks at $k=k_{n}$ in the solid line, which indicate photon absorption and emission, while the dashed line does not show such peaks.

ergy in 2DEG systems. The incident wave packet defined by Eq. (2.2) has an initial width of $\delta=1.2 \mu \mathrm{m}$ and an initial location at $X=-5.11 \mu \mathrm{m}$, which is sufficiently far from the $\mathrm{dc}$ and ac potentials. This width of the wave packet corresponds to a temperature of $T=1.4 \mathrm{~K}$ in actual experimental situations. The size of the whole system is $41.84 \mu \mathrm{m}$. The ac potential is given by Eq. (3.6). The magnitude and width of the ac potential are chosen as $V_{\mathrm{ac}}^{0}=10 \mathrm{meV}$ and $\xi_{\mathrm{ac}}=3000 \AA$, respectively.

The symbol $\omega_{\mathrm{cl}}$ in Fig. 5 denotes the frequency below which the oscillating potential $V_{\mathrm{ac}}(x, y)$ leads to an adiabatic (classical) effect. That is, the electron transport is governed by the instantaneous value of the total potential $V_{\mathrm{dc}}+V_{\mathrm{ac}}{ }^{44}$ This frequency is related to the traversal time $\tau_{\xi_{\mathrm{ac}}}$, which is the time for the electrons to go through the electromagnetic field region, by $\omega_{\mathrm{cl}}=2 \pi / \tau_{\xi_{\mathrm{ac}}}$. The time $\tau_{\xi_{\mathrm{ac}}}$ can be estimated by using the Büttiker-Landauer formula ${ }^{44^{c}}$

$$
\begin{aligned}
\tau_{\xi_{\mathrm{ac}}}= & \sqrt{2 m^{*}} \int_{x_{c}}^{\xi_{\mathrm{ac}} / 2} \frac{d x}{\sqrt{E_{\mathrm{F}}-V_{\mathrm{dc}}(x)}} \\
& +\left(\frac{m^{*}}{2}\right)^{1 / 2} \int_{-x_{c}}^{x_{c}} \frac{d x}{\sqrt{V_{\mathrm{dc}}(x)-E_{\mathrm{F}}}}
\end{aligned}
$$

where $x_{c}$ is the classical turning point defined by $V_{\mathrm{dc}}\left(x_{c}\right)=E_{\mathrm{F}}$. We neglect the small amount of energy fluctuations due to the finite width of the wave packet. Using the above parameters and the function $V_{\mathrm{dc}}(x)$ given by Eq. (3.7), we have $\tau_{\xi_{\mathrm{ac}}}=3.55 \mathrm{ps}$, and thus $\omega_{\mathrm{cl}}=1.13 \mathrm{meV}$. From Fig. 5, enhancements of the transmission probabilities at $\omega \gg \omega_{\mathrm{cl}}$ (the quantum effect region) are small. This is expected because of the loose confinement of the ac field. Figure 6 shows that the enhancements at $\omega>\omega_{\mathrm{cl}}$ indeed come from photon absorption as a quantum effect. The solid line in Fig. 6 represents the square of the Fourier transform of the wave function at $t=39.9 \mathrm{ps}$. The frequency of the external ac field is $\omega=2.0 \mathrm{meV}$. The main peak at $k_{0}=0.22$ (in units of $\pi / \Delta_{x}$, where $\Delta_{x}=65 \AA$ ) corresponds to the transmitting 
wave at energy $E_{\mathrm{F}}$, i.e., $k_{0}=\sqrt{2 m^{*} E_{\mathrm{F}}}$. There are sharp peaks at $k_{n}=\sqrt{2 m^{*}\left(E_{\mathrm{F}}+n \hbar \omega\right)}$, which implies that the small enhancements at $\omega>\omega_{\mathrm{cl}}$ in Fig. 5 are caused by the photon absorption.

The larger enhancement at $\omega \ll \omega_{\text {cl }}$ produced by the adiabatic effect is because the quasistatic potential $\left(V_{\mathrm{dc}}+V_{\mathrm{ac}}\right)$ increases the transmission probability if $T_{\mathrm{dc}}<0.5$, which corresponds to $E_{F}<V_{\mathrm{dc}}$. Under this condition, a low-frequency sinusoidal modulation will produce an instantaneous barrier with a height ranging from $V_{\mathrm{dc}}-V_{\mathrm{ac}}$ to $V_{\mathrm{dc}}+V_{\mathrm{ac}}$. Consequently, the transmission probability can be enhanced during the half-cycle in which the total potential is below $V_{\mathrm{dc}}$. The dashed line in Fig. 6 shows the enhancements of transmission probabilities at low frequencies $\left(\omega / \omega_{\mathrm{cl}} \ll 1\right)$ are due to the adiabatic effect. The dashed line is obtained under the same conditions as those for the solid line, except for the frequency of $\omega=0.1 \mathrm{meV}$. There are no photon peaks indicating the quantum effect. The broad two hills at $k=0.20$ and 0.24 show that the wave packets decelerated and accelerated by the slowly oscillating ac potential, respectively.

\section{PHOTON-ASSISTED QUANTUM TRANSPORT IN DOUBLE-BARRIER STRUCTURES}

As we have shown in Sec. III, it is difficult to achieve quantum photonic effects in electron transports through single-barrier structures. It has also been clarified that either electrons in bound states or spatially confined electromagnetic fields are necessary to obtain a strong coupling between electrons and photons. This understanding suggests that we can utilize the localized nature of quasibound states in a double-barrier structure to achieve the photonic effect. The corresponding long lifetime of the quasibound states (characterized by narrow energy widths in the transmission coefficient peaks) means a long dwell time $\tau$ in the transmission process. Both the long dwell time (at $\tau \gg 1 / \omega$ ) and the bound-state character of the quasibound states are preferred in order to obtain photonic effects.

If the configuration of the double-barrier structure is symmetric, the transmission probability at the quasibound-state energy is exactly unity. Thus any photonic effects on the electron transport will decrease the transmission probability. Since we are mostly interested in an enhancement of transmission probabilities by photon-assisted quantum transport, asymmetric double-barrier structures are more illustrative for our purpose. For asymmetric double-barrier structures, transmission probabilities are not unity even at the energies of resonant states. Thus enhanced transmission could be obtained by applying an ac electromagnetic field. We have chosen the geometries of the asymmetric double barriers such that complete simulations can be accomplished within a reasonable CPU time. For example, if the barrier has a Gaussian shape, then the energy width $\Delta E_{q}$ becomes extremely small for quasibound states far below the barriers. This is because the thickness of the Gaussian potential increases rapidly at its foot. To avoid such a technical difficulty, we have chosen the double-barrier potential structure in the following form:

$$
V_{\mathrm{dc}}(x)=V_{\mathrm{dc}}^{\mathrm{L}} \exp \left[-\frac{\left(x+x_{0}\right)^{4}}{\xi_{\mathrm{L}}^{4}}\right]+V_{\mathrm{dc}}^{\mathrm{R}} \exp \left[-\frac{\left(x-x_{0}\right)^{4}}{\xi_{\mathrm{R}}^{4}}\right] .
$$

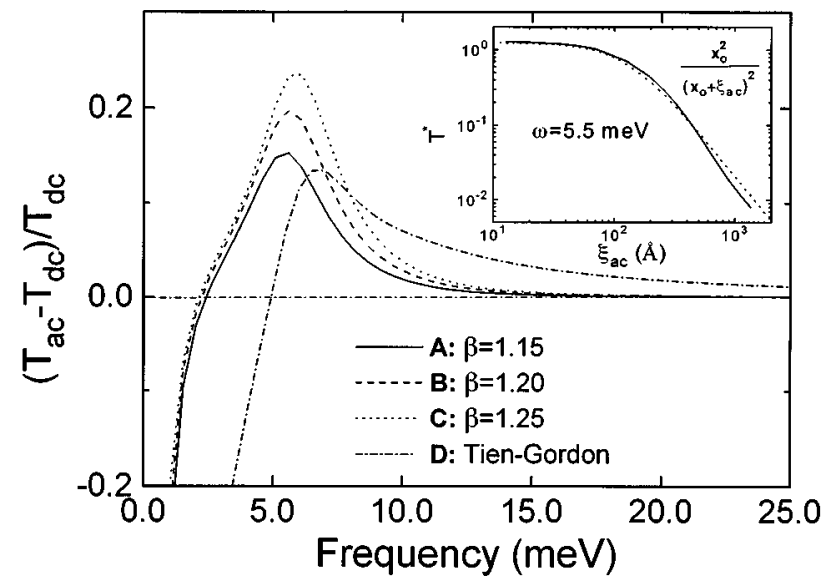

FIG. 7. Frequency dependence of the excess transmission probabilities for asymmetric double-barrier structures with different degrees of asymmetry. The parameters for calculations are presented in the text. The inset shows the radiation-induced excess transmission probability as a function of the width of the electromagnetic field. This has an asymptotic form of $x_{0}^{2} /\left(x_{0}+\xi_{\mathrm{ac}}\right)^{2}$ which is shown by the dotted line with $x_{0}=130 \AA$.

These barriers are "thinner" than the Gaussian profile. The incident wave packet and the applied ac potential are given by Eqs. (2.2) and (3.6), respectively.

Figure 7 shows the frequency dependence of the enhanced or reduced transmission probabilities $T^{*}=\left(T_{\mathrm{ac}}-T_{\mathrm{dc}}\right) / T_{\mathrm{dc}}$, where $T_{\mathrm{ac}}$ and $T_{\mathrm{dc}}$ are transmission probabilities for the system with and without the ac potential. The parameters for the static double-barrier defined by Eq. (4.1) are chosen as follows: $\xi_{\mathrm{L}}=118.4 \AA, \quad \xi_{\mathrm{R}}=204.8 \AA, 2 x_{0}=377.6 \AA$, and $V_{\mathrm{dc}}^{\mathrm{L}}=10.19 \mathrm{meV}$ as common parameters for systems $A, B$, and $C$ (corresponding to lines $A, B$, and $C$ in Fig. 7), respectively; and $V_{\mathrm{dc}}^{\mathrm{R}}=11.72 \mathrm{meV}$ (for $A$ ), $12.23 \mathrm{meV}$ (for $B$ ), and $12.74 \mathrm{meV}$ (for $C$ ). The widths of barriers are small compared to the actual experimental situations, which will yield a dwell time too long for our simulation studies. However, the results shown here illustrate the main features semiquantitatively. Actual experiments should yield even stronger quantum effects due to narrower quasibound levels. In each of these systems, there is only one quasibound state. The quasibound energies $E_{q}$ are $8.91,9.19$, and $9.27 \mathrm{meV}$, and the widths $\Delta E_{q}$ are $0.55,0.61$, and $0.71 \mathrm{meV}$ for systems $A, B$, and $C$, respectively. The parameters for the incident wave packet defined by Eq. (2.2) were chosen as $X=-2.7 \mu \mathrm{m}$ and $\delta=4800 \AA$ commonly for all the three systems, and $E_{\mathrm{F}}=E_{q}$ for each system (namely, $E_{\mathrm{F}}=8.91$, 9.19 , and $9.27 \mathrm{meV}$ for systems $A, B$, and $C$, respectively). The value of $\delta$ corresponds to a temperature of $T=3.3 \mathrm{~K}$. The applied ac potentials are the same for all the systems and are given by Eq. (3.6) with $V_{\mathrm{ac}}^{0}=2.04 \mathrm{meV}$ and $\xi_{\mathrm{ac}}=320.0$ $\AA$. The system size, space division, and time step are 10.48 $\mu \mathrm{m}, 3.2 \AA$, and $12.39 \mathrm{fs}$, respectively, in all the calculations. These three systems have different degrees of asymmetry characterized by the height asymmetry ratio $\beta \equiv V_{\mathrm{dc}}^{\mathrm{R}} / V_{\mathrm{dc}}^{\mathrm{L}}$ The values of $\beta$ are $1.15,1.20$, and 1.25 for systems $A, B$, and $C$, respectively. Calculated dc transmission probabilities $T_{\mathrm{dc}}$ are $0.2382,0.1822$, and 0.1371 at $E=E_{q}$ for systems $A, B$, and $C$, respectively. Line $D$ shows the quantity $T^{*}$ 
calculated using the Tien-Gordon formalism. ${ }^{3}$ In this formalism, the process of photon-assisted tunneling can be modeled with an effective transmission coefficient, $T_{\mathrm{ac}}^{*}=\sum_{n=-\infty}^{\infty} J_{n}^{2}(\alpha) T_{\mathrm{dc}}\left(E_{\mathrm{F}}+n \hbar \omega\right)$, where $J_{n}$ is the $n$th Bessel function, $\alpha=V_{\mathrm{ac}}^{*} / \hbar \omega$, and $V_{\mathrm{ac}}^{*}$ is the amplitude of the effective potential. The dc transmission probability $T_{\mathrm{dc}}(E)$ is calculated from the conventional transfer-matrix method. This calculation has been used to analyze experimentally observed photon-induced current in vertical and lateral quantum-well structures. ${ }^{11,19}$ The justification of using this simple picture lies in the fact that the photon absorption and emission mainly occur within the well, where the dipolemoment integration yields nonvanishing results. Consequently, the only effect of radiation is a modification of the outgoing electron wave functions, which can be modeled using an effective transmission coefficient $T_{\text {ac }}^{*}$. For line $D$, we chose the value of $V_{\mathrm{ac}}^{*}$ to be $3.06 \mathrm{meV}\left(=1.5 V_{\mathrm{ac}}^{0}\right)$ and all the other parameters to be the same as those for line $C$. The result qualitatively resembles line $C$. However, similar to Fig. 1, $T_{\mathrm{dc}}(E)$ calculated from the transfer-matrix method has a sharper peak than that calculated using the numerical path-integral method. Thus the radiation-induced transmission coefficients calculated from the two methods do not agree with each other quantitatively.

It should be noted that our numerical results show only quantum effects in the frequency region investigated. The crossover frequency $\omega_{\mathrm{cl}}$ to the adiabatic (classical) effect region is approximately equal to $\Delta E_{q}$, which are the lowest frequencies in our calculations.

Let us first discuss the result for system $A$, which is indicated by the solid line. For the lowest-frequency region, the energy of the excited electron is slightly shifted from the resonant level $E_{q}$. Thus the transmission probability becomes smaller than the value of the dc transmission probability $T_{\mathrm{dc}}$ at $E_{\mathrm{F}}=E_{q}$. By increasing the frequency of the ac field, the electron-photon coupling decreases for the same reason as for the decrease of the escape probability at high frequencies in Fig. 2. Therefore, the transmission probability rapidly approaches the value of $T_{\mathrm{dc}}$. By increasing the frequency further from the value $\omega_{s}=V_{\mathrm{dc}}^{\mathrm{R}}-E_{\mathrm{F}}=2.81 \mathrm{meV}$, the energy of the excited electron exceeds the height of the rightside barrier. Thus the transmission probability increases beyond $T_{\mathrm{dc}}$, resulting from a photon-assisted over-the-barrier transport. In the highest-frequency region, the interaction becomes too weak to have any photonic effects, and thus the transmission probability approaches $T_{\mathrm{dc}}$ asymptotically. Consequently, $T^{*}$ has a peak at $\omega_{0} \sim \omega_{s}$.

The dashed and dotted lines, which indicate the results for systems $B$ and $C$, show that the values of $T_{\max }^{*}$ increase with $\beta$. This means a stronger asymmetry yields a larger photonic effect. This is because $T_{\mathrm{dc}}$ at $E_{q}$ is smaller for a more asymmetric double-barrier structure, while $T_{\mathrm{ac}}$ is approximately constant for $\omega>\omega_{s}$. Thus the enhanced $T^{*}=\left(T_{\mathrm{ac}}\right.$ $\left.-T_{\mathrm{dc}}\right) / T_{\mathrm{dc}}$ is greater for a greater $\beta$. In order to clarify the confinement effect of the ac field quantitively, the inset of Fig. 7 illustrates the maximum value $T_{\max }^{*}$ as a function of the width $\xi_{\mathrm{ac}}$ of the ac field. The conditions for this calculation are the same as those for line $B$, except that $\xi_{\text {ac }}$ is now a variable and $\omega=5.5 \mathrm{meV}$. This frequency is chosen because it is the frequency at which $T^{*}$ takes its maximum value $T_{\max }^{*}$. The enhanced transmission probability $T_{\max }^{*}$ is approximately constant at small values of $\xi_{\mathrm{ac}}$, and decreases algebraically with an increase of $\xi_{\mathrm{ac}}$ when $\xi_{\mathrm{ac}}>x_{0}$. The $T^{*}-\xi_{\mathrm{ac}}$ relation has an approximate functional form of $x_{0}^{2} /\left(x_{0}+\xi_{\mathrm{ac}}\right)^{2}$. This implies that the decrease is simply due to a decrease of the ac electric-field strength in the active region between the two barriers. This feature differs qualitatively from that in Fig. 4, which shows the strength of the coupling between a free electron and a confined electromagnetic field. In that case, the interaction vanishes exponentially as $\xi_{\mathrm{ac}}$ increases because of the selection rule.

\section{CONCLUSIONS}

In summary, we have performed numerical studies on the effect of photon-assisted quantum transport in order to obtain conditions for substantial enhancements of transmission probabilities. These conditions require a spatial localization of either the electronic wave function or the applied electromagnetic fields. The validity of these conditions were quantitatively demonstrated by two numerical simulations. The first one is the frequency dependence of the photondetaching probability of electrons from a bound state. The result exhibits a strong coupling between the electron and the ac field, even if the ac field is not spatially confined. It was also clarified that the quantum coupling becomes weak as the frequency of the ac field increases. The second example is the interaction between a free electron and a spatially confined electromagnetic field. We have investigated how the strength of the interaction depends on the width of the ac field. The result shows that a confinement of the ac field within a region narrower than the wavelength of the excited electron is necessary to obtain strong coupling between free electrons and electromagnetic fields. Consequently, photonassisted transport in single-barrier structures will be difficult to achieve if the photon field is spatially extended. This understanding may explain why photon-assisted transport has not been observed in single-barrier devices such as quantum point contacts. One alternative approach to achieve a strong electron-photon coupling is to use double- or multiple-barrier structures to create quasibound states. The spatially localized nature of these states will provide the momentum spread necessary for the photon excitation process. Our simulation studies on asymmetric double-barrier structures have yielded a positive effect of photon-assisted transport, even with spatially extended photon fields.

\section{ACKNOWLEDGMENTS}

We would like to thank P. Maass and T. Nakayama for stimulating discussions. Numerical calculations were performed on the FACOM VPP500 of Supercomputer Center, Institute for Solid State Physics, University of Tokyo. One of the authors (K.Y.) acknowledges fellowship support for a research investigator abroad provided by the Ministry of Education, Science and Culture, Japan. This work was supported by the ONR under Grant No. N00014-90-J-1829 at UCLA, and by the NSF under Grant No. 94-00334DMR at MIT. 
*Deceased.

${ }^{1}$ Q. Hu, Appl. Phys. Lett. 62, 837 (1993).

${ }^{2}$ S. Feng and Q. Hu, Phys. Rev. B 48, 5354 (1993).

${ }^{3}$ P. K. Tien and J. P. Gordon, Phys. Rev. 129, 647 (1963).

${ }^{4}$ R. A. Wyss, C. C. Eugster, J. A. del Alamo, and Q. Hu, Appl. Phys. Lett. 63, 1522 (1993).

${ }^{5}$ R. A. Wyss, C. C. Eugster, J. A. del Alamo, Q. Hu, M. J. Rooks, and M. R. Melloch, Appl. Phys. Lett. 66, 1144 (1995).

${ }^{6}$ W. A. de Heer, Rev. Mod. Phys. 65, 611 (1993).

${ }^{7}$ C. Meier and V. Engel, Phys. Rev. Lett. 73, 3207 (1994).

${ }^{8}$ H. Katori and F. Shimizu, Phys. Rev. Lett. 73, 2555 (1994).

${ }^{9}$ K. Hino, P. M. Bergstrom, and J. H. Macek, Phys. Rev. Lett. 72, 1620 (1994).

${ }^{10}$ See references in Intersubband Transitions in Quantum Wells, Vol. 288 of NATO Advanced Study Institute, Series B: Physics, edited by E. Rosencher, B. Vinter, and B. Levine (Plenum, New York, 1992).

${ }^{11}$ P. S. S. Guimaraes, B. J. Keay, J. P. Kaminski, S. J. Allen, P. F. Hopkins, A. C. Gossard, L. T. Florez, and J. P. Harbison, Phys. Rev. Lett. 70, 3792 (1993).

${ }^{12}$ A. H. Dayem and R. J. Martin, Phys. Rev. Lett. 8, 246 (1962).

${ }^{13}$ I. P. Nevirkovets, L .P. Stryzhko, and A. V. Poladich, Physica B 194-196, 2395 (1994).

${ }^{14}$ M. Cirillo, I. Modena, F. Santucci, P. Carelli, M. G. Castellano, and R. Leoni, J. Appl. Phys. 73, 8637 (1993).

${ }^{15}$ Q. Hu, C. A. Mears, P. L. Richards, and F. L. Lloyd, Phys. Rev. B 42, 10250 (1990).

${ }^{16}$ L. P. Kouwenhoven, S. Jauhar, J. Orenstein, P. L. McEuen, Y. Nagamune, J. Motohisa, and H. Sakaki, Phys. Rev. Lett. 73, 3443 (1994).

${ }^{17}$ L. P. Kouwenhoven, S. Jauhar, K. McCormick, D. Dixon, P. L. McEuen, Yu V. Nazarov, N. C. van der Vaart, and C. T. Foxon, Phys. Rev. B 50, 2019 (1994).

${ }^{18}$ C. Karadi, J. Opt. Soc. Am. B 11, 2566 (1994).

${ }^{19}$ S. Verghese, R. A. Wyss, Th. Schaepers, A. Foerster, M. J. Rooks, and Q. Hu, Phys. Rev. B 52, 14834 (1995).

${ }^{20}$ L. Esaki and R. Tsu, IBM J. Res. Dev. 14, 61 (1970).

${ }^{21}$ R. Tucker, IEEE J. Quantum Electron. QE-15, 1234 (1979).

${ }^{22}$ D. S. Pan and C. C. Mong, J. Appl. Phys. 61, 2082 (1987).

${ }^{23}$ T. C. L. G. Sollner, W. D. Goodhue, P. E. Tanneuwald, C. D.
Parker, and D. D. Peck, Appl. Phys. Lett. 43, 588 (1983).

${ }^{24}$ T. C. L. G. Sollner, E. R. Brown, W. D. Goodhue, and H. Q. Le, Appl. Phys. Lett. 50, 332 (1987).

${ }^{25}$ E. R. Brown, T. C. L. G. Sollner, C. D. Parker, and W. D. Peck, Appl. Phys. Lett. 55, 1777 (1989).

${ }^{26}$ W. Cai, T. F. Zheng, P. Hu, M. Lax, K. Shum, and R. R. Alfano, Phys. Rev. Lett. 65, 104 (1990).

${ }^{27}$ V. A. Chitta, R. E. M. de Bekker, J. C. Maan, S. J. Hawksworth, J. M. Chamberlain, M. Henini, and G. Hill, Semicond. Sci. Technol. 7, 432 (1992).

${ }^{28}$ V. A. Chitta, C. Kutter, R. E. de Bekker, J. C. Maan, S. J. Hawksworth, J. M. Chamberlain, M. Henini, and G. Hill, J. Phys. Condens. Matter 6, 3945 (1994).

${ }^{29}$ H. Buhmann, J. Wang, L. Mansouri, P. H. Beton, L. Eves, M. Heath, and M. Henini, Solid State Electron. 37, 973 (1994).

${ }^{30}$ N. Lebedev, P. Maass, and S. Feng, Phys. Rev. Lett. 74, 1895 (1995).

${ }^{31}$ J. Crank and P. Nicolson, Proc. Cambridge Philos. Soc. 32, 50 (1947).

${ }^{32}$ G. G. O’Brien, M. A. Hyman, and S. Kaplan, J. Math. Phys. 29, 223 (1951).

${ }^{33}$ See, for example, W. F. Ames, Numerical Methods for Partial Differential Equations (Academic, New York, 1977).

${ }^{34}$ A. Askar and A. S. Cakmak, J. Chem. Phys. 68, 2794 (1978).

${ }^{35}$ E. A. McCullough and R. E. Wyatt, J. Chem. Phys. 54, 3578 (1978).

${ }^{36}$ M. D. Feit, J. A. Fleck, and A. Steiger, J. Comput. Phys. 47, 412 (1982).

${ }^{37}$ D. Kosloff and R. Kosloff, J. Comput. Phys. 52, 35 (1983).

${ }^{38}$ R. Viswanathan, S. Shi, E. Vilallonga, and H. Rabitz, J. Chem. Phys. 91, 2333 (1989).

${ }^{39}$ R. Feynman and A. R. Hibbs, Quantum Mechanics and Path Integrals (McGraw-Hill, New York, 1965).

${ }^{40}$ W. Gordon, Z. Phys. 40, 117 (1927).

${ }^{41}$ D. M. Volkov, Z. Phys. 94, 250 (1935).

${ }^{42}$ R. Grobe and M. V. Fedorov, J. Phys. B 26, 1181 (1993).

${ }^{43}$ L. D. Landau and E. M. Lifshitz, Quantum Mechanics: Nonrelativistic Theory (Pergamon, New York, 1991).

${ }^{44}$ M. Büttiker and R. Landauer, Phys. Rev. Lett. 49, 1739 (1982). 